\title{
Engineered Human Umbilical Vein Endothelial Cells AB-205
}

National Cancer Institute

\section{Source}

National Cancer Institute. Engineered Human Umbilical Vein Endothelial Cells AB-205. NCI

Thesaurus. Code C162248.

A population of ex vivo expanded, genetically eng ineered CD31 (platelet endothelial cell adhesion molecule; PECAM-1)-positive human umbilical vein endothelial cells (HUVECs) derived from human umbilical vein tissue, that can be used to enhance the hematopoietic stem and progenitor cells (HSPCS) transplantation potential and improve blood cell recovery. Following autologous stem cell transplantation (ASCT) and upon the administration of the eng ineered HUVEC AB-205, the endothelial cells secrete angiocrine growth factors and interact with the HSPCs, thereby forming endothelial cell network structures and improving engraftment potential. AB-205 also interacts with injured or damaged vascular niche cells, thereby promoting blood cell recovery and improving tissue regeneration. This enhances recovery from toxicities related to chemo/radiation regimens. 